\title{
Dual-polarized dipole radiation elements for the modern base station antennas
}

\author{
Vladislav Komov ${ }^{1, *}$, Victor Sledkov ${ }^{1}$, and Li ZiMeng ${ }^{1}$ \\ ${ }^{1}$ Guandong Sigtenna Communication Technology Co., Ltd., Jin da Automobile logistic city, No.4, \\ Yanwo village, Shipai Town, Dongguan, China
}

\begin{abstract}
Two improved designs of wideband dual polarization antennas for base station. The first design is the crossed dipoles for the frequency bands 1695-2695 MHz. The presented shape of crossed dipole provides a low level of crosspolarization, VSWR better than 1.4 , the isolation between polarizations is better than $-33 \mathrm{~dB}$ and the beam width in the horizontal plane is $65 \pm 4$ degrees. The second design is the square dipole in the frequency range $690-960 \mathrm{MHz}$ provides the beam width in the horizontal plane $60 \pm 5$ degrees, E-plane $58 \pm 3$ degrees, H-plane $64 \pm 3$ degrees, the isolation between polarizations better than $-35 \mathrm{~dB}$.
\end{abstract}

\section{Introduction}

In these days of high mobile phone usage, market needs huge quantity of wideband dual polarized antennas each year therefore considerable work has been done in this field to create such antennas which are easy to be manufactured. The most of antennas for base station have to provide 65 degree beam width, good cross polarization discrimination and matching with feeding cables through wide frequency band. Crossed dipoles supported by a balun are the simplest dual polarized antennas therefore hundreds of such antennas were invented to extend its frequency band and decrease cost of manufacturing, however, due to the constant growth in the weight and size requirements of base station antennas, this issue remains relevant. One radiator of this kind described in [1].

And also due to crossed dipoles create too wide beam at $\mathrm{H}$ plane more complicated radiators were invented to decrease beam width. The radiating arrangement containing four dipoles placed above a reflective conductive plate and arranged in a shape of a dipole square. Described a dual slant polarized antenna having approximately 65 degrees half power beam width in horizontal plane that reduces unwanted interference between antenna elements in the array.

The present paper is devoted to research and improvement a wideband directional antenna radiating two linear polarizations and having better cross polarization discrimination and matching with feeding line through a wide frequency band than known antennas, providing less unwanted coupling between neighbor antennas of antenna array for single- and multi-band systems.

\footnotetext{
*Corresponding author: vladislaw.komov2018@yandex.ru
} 


\section{The design of the antennas}

Known crossed dipoles have beam width at magnetic H-plane wider than beam width at electrical E-plane. The biggest difference is at the lowest frequency of operating frequency band. Wide beam at $\mathrm{H}$ plane creates low cross polarization discrimination and unwanted coupling between neighbor antennas of antenna's arrays. Therefore it is need to reduce beam width at $\mathrm{H}$ plane to improve cross polarization discrimination and decrease coupling between neighbor antennas of antenna's array. At the same time it is need to match antenna with feeding cables.

The preferred option according to the results of research the optimal form of the radiating shape for frequency band $1695-2695 \mathrm{MHz}$ is shown in Fig 1(a), (b). The radiating arrangement and the feeding element consisting of two feeding lines and two perpendicular balun having a common base placed on the reflective conductive plate and connected with the radiating arrangement by its top ends. The radiating arrangement consists of four arms placed in a plane in parallel to the reflective conductive plate and separated from each other by gaps. Each arm comprises the outer conductor forming a hollow outer counter of the arm and three additional conductors placed inside of a hollow outer counter and connected by its ends together and with the outer conductor. The outer conductor consists of two straight portions connected to the balun by the first ends and two portions in a shape of arc connected together and with straight portions. The first additional conductor of a straight shape is connected to the outer conductor between two arcs and two other additional conductors in a shape of two arcs are connected to outer conductor close by the second ends of straight portions. The main difference described structure with the known designs of crossed dipoles is width of the arm $\mathrm{W}$ is bigger than half width $\mathrm{A}$ of the radiating arrangement that provides less difference between slant planes of beam width $\mathrm{E}$ and $\mathrm{H}$ is shown in Fig 1(b).

Feeding element contains longitudinal holes inside of balun where placed inner conductors of feeding lines connected to the radiating arrangement by conductive bridges are shown in Fig 1(a).

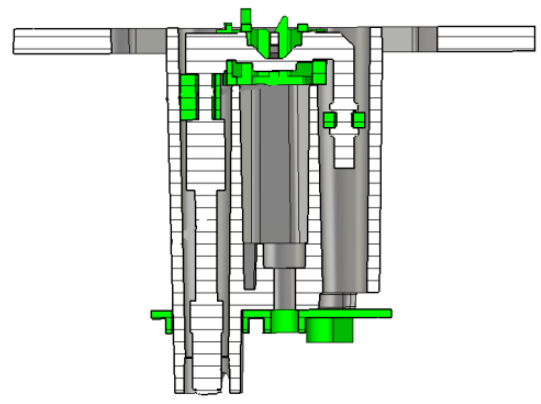

(a)

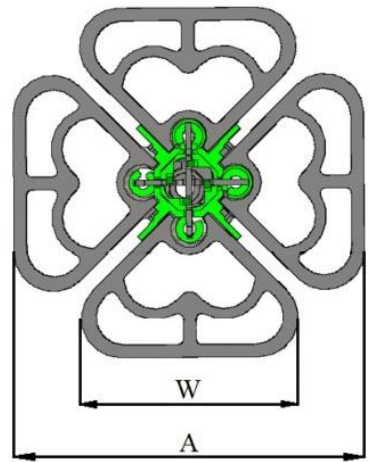

(b)

Fig. 1. (a) cross section of the crossed dipole, (b) top view of the crossed dipole.

Starting from the prior art mentioned, it is therefore the first object of the present invention shown in Fig 2 (a),(b) to create a dual-polarized antenna providing 60-65 degree half power beam width and cross polarization ratio better $-10 \mathrm{~dB}$ at edges of $+/-60$ degree sector through at least $46 \%$ frequency band. The second object is to improve matching of a dual-polarized antenna operating through a wide frequency band. The third object is to create a wide band antenna which is easy to be connected to a beam forming network. The fourth object is to create a low band antenna for dual band antenna arrays, with the result 
that has less height and creates less distortions of a beam of a high band radiator placed at the middle of a low band radiator.

A dual polarized antenna comprising the radiating arrangement containing four dipoles placed above a reflective conductive plate and arranged in a shape of a dipole square. Arms of each dipole are connected to the top ends of two conductive members and the bottom ends of conductive members are connected to a common base placed on a reflective conductive plate. Dipole arms contain a flat surface placed in parallel to the reflective ground plate and projections placed on the edges of the arm and directed towards the reflective ground plate. The additional conductive member is placed along arms of each dipole and fixed to dipole arms by supporting plastic member. The ends of the additional conductive member are bent towards dipole arms and separated from ends of dipole arms by thin dielectric films is shown in Fig 2(a),(b). The bottom ends of central line of the feed part directly connected to the strip lines of feeding networks formed on a printed circuit board placed on the common base that creates the possibility of adjustment of matching feed line with radiation part and reduce high of dipole.

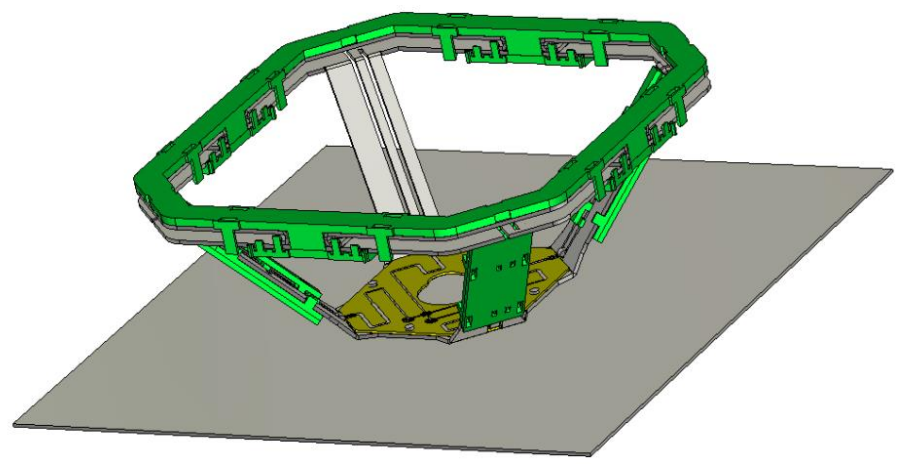

(a)



(b)

Fig. 2. Appearance of the square dipole (a) perspective view, (b) placed of additional conductivity members of the dipole.

\section{Results of simulation}

The electromagnetic analysis of this structures made by CAD. The results of simulation presented in Fig.3-5. Table.1. 


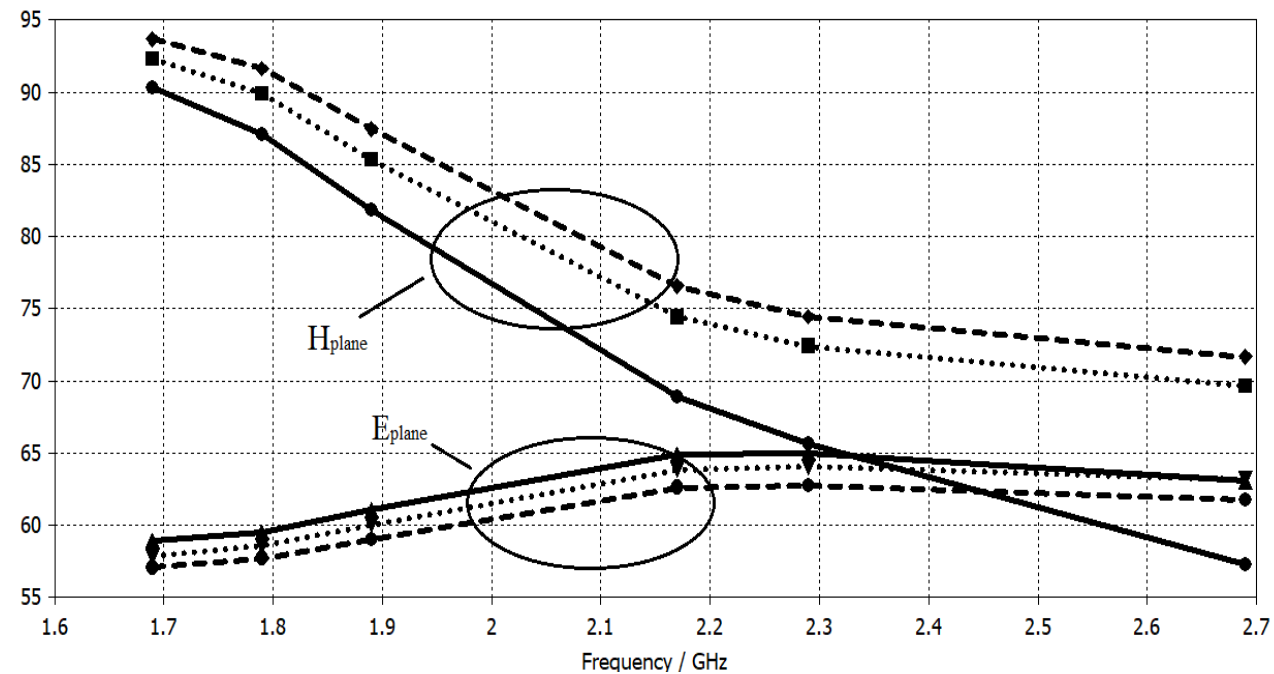

(a)

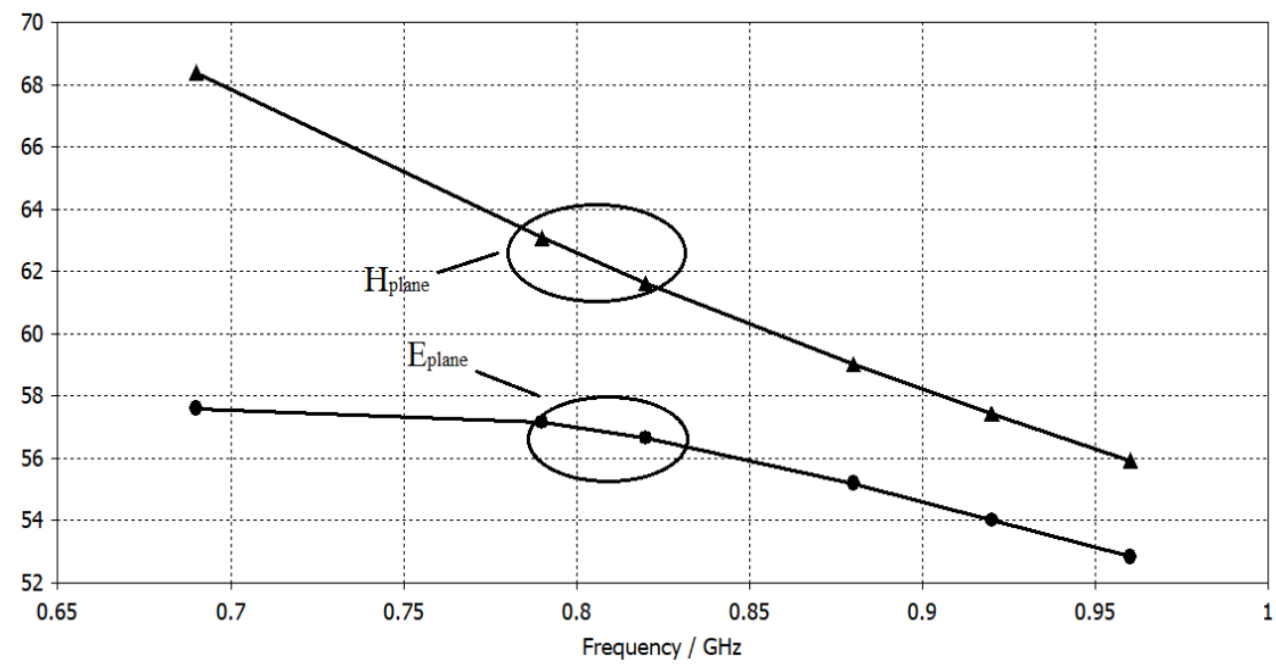

(b)

Fig. 3. Calculated dependences half power beam width in at $\mathrm{H}$ plane and at $\mathrm{E}$ plane via frequency (a) increasing the width of the crossed dipole arm to $\mathrm{W}>\mathrm{A} / 2$ for $1695-2695 \mathrm{MHz}$, (b) Square dipole for 690-960 MHz.

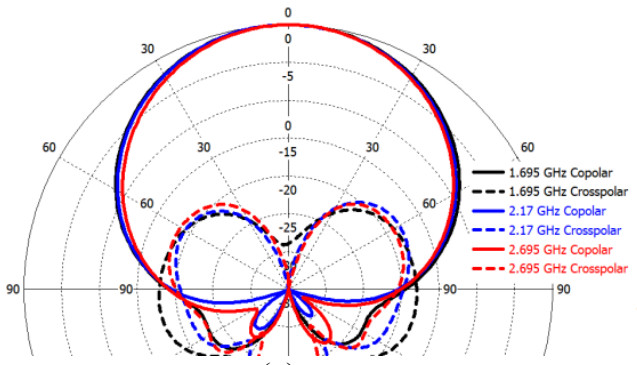

(a)

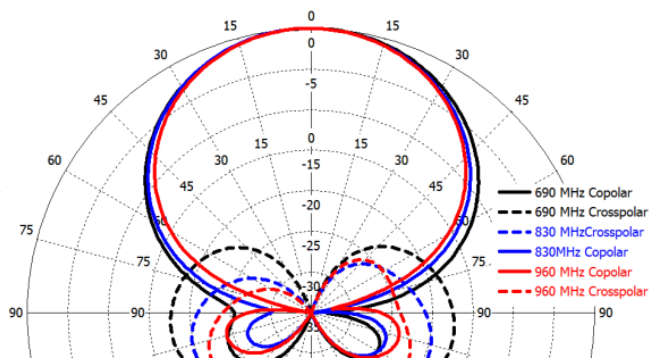

(b)

Fig. 4. Results of simulations co-polar and cross-polar patterns at horizontal plane of described dualpolarized antennas, (a) crossed dipole $1695-2695 \mathrm{MHz}$, (b) square dipole 690-960 MHz. 


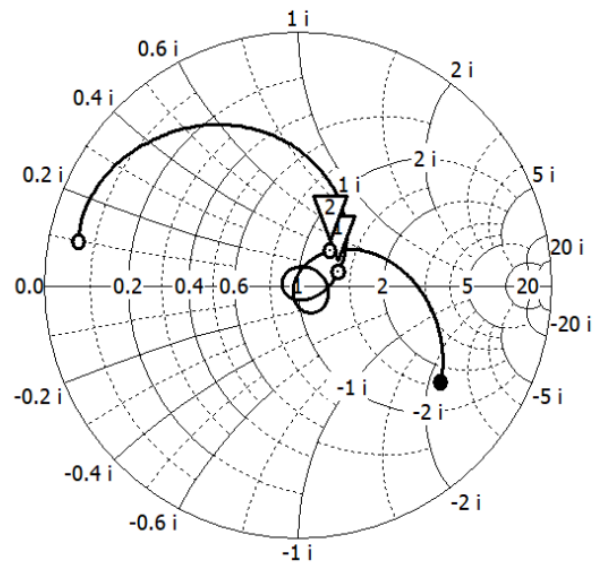

(a)

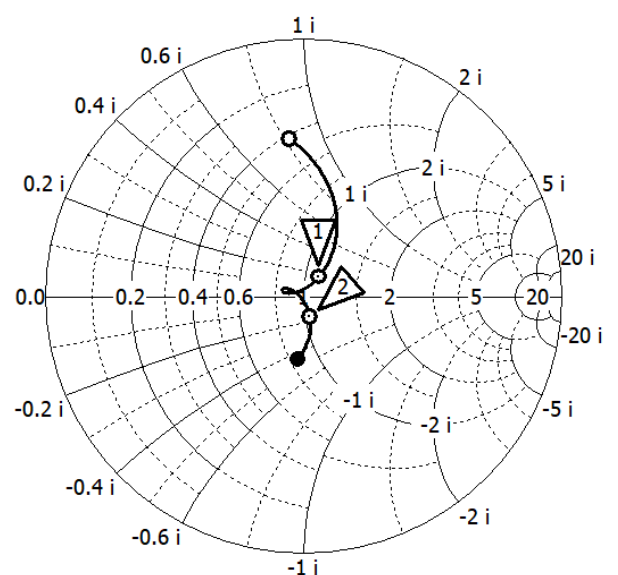

(b)

Fig. 5. The Smith Chart of the described dual-polarization antennas, (a) crossed dipole 1695-2695 $\mathrm{MHz}$, (b) square dipole 690-960 MHz.

Table.1. Beam width values of the presented designs

\begin{tabular}{|l|c|c|}
\hline Frequency band [MHz] & $\begin{array}{l}1695-2695 \\
\text { Crossed dipole }\end{array}$ & $\begin{array}{l}690-960 \\
\text { Square dipole }\end{array}$ \\
\hline $\begin{array}{l}\text { The beam width in the } \\
\text { horizontal plane at the } \\
\text { level of -3 dB }\end{array}$ & $65 \pm 4$ & $60 \pm 5$ \\
\hline $\begin{array}{l}\text { Beam width in the E- } \\
\text { plane at the level -3 dB }\end{array}$ & $62 \pm 3$ & $58 \pm 3$ \\
\hline $\begin{array}{l}\text { Beam width in the H- } \\
\text { plane at the level -3 dB }\end{array}$ & $75 \pm 15$ & $64 \pm 5$ \\
\hline \multicolumn{1}{|c|}{ VSWR between } & $>31.39$ & $\geq 1.35$ \\
\hline $\begin{array}{l}\text { Isolation } \\
\text { polarizations }\end{array}$ & $>35 \mathrm{~dB}$ \\
\hline
\end{tabular}

Calculated dependences show the shape of radiating arrangement according to description significantly decreases difference between half power beam width at $\mathrm{H}$ plane and at E plane via operating frequency band $1695-2695 \mathrm{MHz}$ and provides equal half power beam width at frequency $2300 \mathrm{MHz}$.

\section{Conclusion}

The described designs easy to be manufactured and provides good matching with feeding line and high cross polarization discrimination at least through $46 \%$ frequency band. Both structures due to narrow beam width at slant planes $\mathrm{E}$ and $\mathrm{H}$ possible to use in different single- and multi-band systems where antennas placed close to each other.

\section{References}

1. Patent $C N 204424435 U$ 